\title{
Correntes de pensamento nacionais sobre sistematização da assistência de enfermagem
}

Concepts associated to systematization of nursing care in Brazilian journals

Conceptos asociados a la sistematización de la asistencia de enfermaria en la literatura brasileña

\author{
Patrícia dos Santos Claro Fuly', Joséte Luzia Leite', Suzinara Beatriz Soares Lima'
}

'Universidade Federal do Rio de Janeiro. Escola de Enfermagem Ana Nery. Rio de Janeiro, RJ

Submissão: 23/07/2007

Aprovação: 08/10/2008

\section{RESUMO}

Trata-se de uma pesquisa Que tem por objetivo analisar a produção bibliográfica sobre a sistematização da assistência de enfermagem a fim de discutir conceitos associados ao tema. Foram analisados livros e artigos nacionais publicados na base de dados da Biblioteca Virtual em Saúde, Medline, Lilacs e Scielo, no período de janeiro de 2000 a janeiro de 2008. Formam analisados 1 I artigos, tendo sido identificadas três correntes de pensamento distintas para definição de termos associados à sistematização da assistência de enfermagem. Tal fato aponta para a dificuldade de articulação, teórico-prática, evidenciada pelos conflitos existentes na própria literatura.

Descritores: Enfermagem; Processos de enfermagem; Organização \& administração.

\section{ABSTRACT}

This study is a research that has as objective analyze the bibliographical production about the Systematization of Nursing Care in order to discuss concepts associated to the subject. Were analyzed books and national articles published in the database of the Virtual Library in Health, Medline, Lilacs and Scielo, in the period of lanuary of 2000 to lanuary of 2008. Were analyzed 11 articles, having been identified three distinct kind of thought to define concepts associated to Systematization of Nursing Care. Such fact aims for the difficulty of theoretical and pratical articulation, evidenced by the conflicts existing in the literature.

Descriptors: Nursing; Nursing process; Organization \& administration.

\section{RESUMEN}

Esta es una investigación que tiene como objetivo el análisis de la producción bibliográfica sobre la sistematización de la asistencia de enfermería con el fin de debatir las ideas relacionadas con el tema. Se analizaron los libros y los artículos nacionales publicados en la base de datos de la Biblioteca Virtual en Salud, en Medline, Lilacs y Scielo, en el período de enero de 2000 a enero de 2008. Se analizaron I I artículos, al Que se ha identificado tres tipos de pensamiento para definir conceptos asociados a la sistematización de la asistencia de enfermería. Tal hecho apunta la dificultad de la articulación teórica y práctica, evidenciada por los conflictos presentes en la literatura. Descriptores: Enfermería; Procesos de enfermería; Organización \& administración. 


\section{INTRODUÇÃO}

Desde o advento dos processos de Acreditação Hospitalar, o discurso em defesa da Sistematização da Assistência de Enfermagem (SAE) e metodologias para implementação do processo de enfermagem em unidades hospitalares, vem ganhando adeptos. Tal movimento cresce tendo em vista Que o emprego de metodologias para a organização do trabalho da enfermagem fazse essencial para obtenção desses certificados de acreditação hospitalar.

No Que se refere à Qualidade, e em especial a Acreditação Hospitalar a muito pouco entendimento destas em relação as ações de enfermagem. Ao analisarmos os critérios para se acreditar uma instituição hospitalar, em todos os níveis e em todas as unidades eles estão relacionados e centralizados na equipe de enfermagem. Há de se ver Que a implementação da SAE é um grande passo para o acreditamento das instituições hospitalares, pois contempla muitos critérios dos instrumentos avaliadores. Assim percebe-se a importância da SAE para o enfermeiro trabalhar, ou seja, organizar seu serviço partindo desta. Daí cabe o Questionamento: será que a SAE direcionaria para indicadores de Qualidade, mesmo não explícitos, de referência na enfermagem? Fazer uma reflexão neste sentido, faz-se necessária, pois o processo de acreditação hospitalar também vislumbra formas de desenvolver o trabalho sistemático de modo que possa ser mensurada por ela.

Nesta perspectiva, na memória histórica da Qualidade, Florence Nightingale (1820-1910), destaca-se como mobilizadora dos princípios e indicadores de Qualidade. Os seus cálculos de taxas de mortalidade, a análise desses dados e a organização, lhe permitiram identificar padrões de assistência Que levaram à melhoria da assistência prestada aos pacientes à época. Observa-se, assim, a tentativa de estabelecimento de modelos de atendimento semelhantes ao processo de acreditação hoje em desenvolvimento $^{(1,2)}$.

Atualmente a implementação da SAE, mais do Que uma opção para a organização do trabalho do enfermeiro, apresenta-se como uma Questão deontológica para a enfermagem. A Resolução COFEN $n^{\circ}$. 272/2002 enfatiza a necessidade de aplicação da sistematização da assistência na prática cotidiana da enfermagem em seus diferentes cenários de trabalho: uma atividade privativa do enfermeiro Que, utiliza método e estratégia de trabalho científico para a identificação das situações de saúde/doença, subsidiando ações de assistência de Enfermagem Que possam contribuir para a promoção, prevenção, recuperação e reabilitação da saúde do indivíduo, família e comunidade $^{(3)}$.

A mesma resolução do COFEN afirma ainda Que cabe ao enfermeiro: A implantação, planejamento, organização, execução e avaliação do processo de enfermagem.

No Rio de Janeiro a SAE por meio da aplicação do processo de enfermagem em todas as suas etapas, ainda é bastante incipiente, como demonstra o estudo de Fuly, Freire \& Almeida realizado em 27 Centros de Terapia Intensiva. A baixa aplicabilidade da SAE no estado ocorre, dentre outros fatores, por um desconhecimento a cerca do processo de enfermagem ${ }^{(4)}$.

Nesse contexto observamos Que existem alguns conceitos associados ao tema sistematização da assistência, Que têm sido empregados de forma conflituosa no vocabulário popular da enfermagem. Processo de enfermagem, metodologia da assistência e a própria sistematização da assistência ora apresentam-se como sinônimos e ora apresentam-se com diferentes definições. Diante dessa perspectiva considera-se que o emprego equivocado desses termos gera conflito e dificuldade de entendimento sobre a prática profissional da enfermagem.

Sendo assim esse estudo tem por objetivo analisar a produção bibliográfica sobre sistematização da assistência; e discutir conceitos associados ao tema, contribuindo para uma reflexão sobre a SAE e sua aplicabilidade em hospitais do Rio de Janeiro.

\section{METODOLOGIA}

Trata-se de uma revisão de literatura onde conceitos foram discutidos, com base em autores de referência na área, contrastando com referências publicadas nas bases de dados MEDLINE, LILACS, SciELO da Biblioteca Virtual em Saúde (BVS).

Para a coleta de dados foram utilizadas as palavras-chave: SISEMATIZAÇÃO DA ASSISTÊNCIA / ENFERMAGEM / GERENCIAMENTO. Foram estabelecidos como critérios de inclusão na amostra: artigos científicos e livros com aderência ao tema gerenciamento de enfermagem Que atendessem ao recorte temporal de 2000 a 2008; os Que apresentassem um enfoque hospitalar e os Que não tratassem de implementação da SAE a situações clínicas em particular.

A revisão de literatura foi realizada nos meses de outubro de 2007 a janeiro de 2008. Foram selecionados 20 artigos, sendo Que destes 11 foram analisados. Também foram analisados 6 livros correlatos à temática.

No recorte temporal foram utilizadas referências desde 2000, pois em 1999, em São Paulo foi instituída uma resolução do $\operatorname{COREN}^{(5)}$ Que tornou obrigatória a implementação da SAE, daí a relevância de considerar artigos publicados logo após essa determinação.

A análise foi realizada por meio do método comparativo, onde os conceitos associados à sistematização da assistência de enfermagem foram analisados.

\section{RESULTADOS}

Essa revisão bibliográfica permitiu identificar uma série de conceitos empregados de forma conflituosa nas literaturas consultadas. Existem atualmente três correntes Que divergem no emprego desses termos nas publicações atuais: a primeira corrente trata os termos Sistematização da Assistência de Enfermagem (SAE), Metodologia da Assistência de Enfermagem (MAE) e Processo de Enfermagem (PE) como termos distintos. A segunda corrente trata Metodologia da Assistência de Enfermagem e Processo de Enfermagem como sendo termos equivalentes. E existe ainda uma terceira corrente afirmando Que os três termos são sinônimos.

Foram encontrados 03 artigos da primeira corrente Que trata os termos SAE, MAE e PE como distintos, 06 da segunda corrente e 02 da terceira corrente. Dos 11 artigos foi selecionado um Que abordava especificamente a Questão do emprego de vocabulário padronizado de enfermagem.

Diante deste fato, a seguir são situados os autores de referência e a literatura identificada de acordo com as três correntes descritas. 
No Que tange a primeira corrente, Que defende a diferenciação entre os três termos, e que constitui aquela Que contém informações com as Quais pactuamos, destaca-se a opinião de alguns autores.

"Sistematização da Assistência de Enfermagem é todo o planejamento registrado da assistência Que abrange desde a criação e implementação do manual de normas e rotinas das unidades à descrição padronizada, até a adoção do Processo de Enfermagem"(6).

A definição das autoras traz uma Questão Que tem sido motivo de muitas discussões para os enfermeiros, a do registro de enfermagem. É sabido Que o registro é fundamental para as atividades, porém considera-se Que o fato do enfermeiro não registrar no prontuário implica numa série de Questões éticas e deontológicas, porém não significa dizer Que em seu processo intelectual ele não sistematizou a assistência.

É importante ressaltar Que o registro é uma atividade fim e não o meio para sistematizar a assistência. A documentação do processo de enfermagem não é o processo de enfermagem por si só, a documentação é um reQuisito legal indispensável à prática, porém o registro no prontuário não é sinônimo de sistematização e sim do cumprimento de uma tarefa importante para a $S A E^{(7)}$.

Nessa linha de pensamento pode-se compreender a Sistematização da Assistência de Enfermagem como a organização do trabalho da enfermagem, Quanto ao método, pessoal e instrumentos, a fim de tornar possível a realização do Processo de Enfermagem $^{(8)}$. A SAE prevê a definição da natureza do trabalho a ser realizado e a definição do processo de enfermagem, desde a base teórico-filosófica, até o tipo de profissional, os métodos, os objetivos e os recursos materiais, para a produção do cuidado ${ }^{(9)}$.

O Processo de Enfermagem tem sido confundido como Método de Assistência de Enfermagem, mas são idéias distintas. Metodologia da Assistência de Enfermagem é um caminho, um modo de conduzir o trabalho com uma lógica, sendo um dos elementos da $\operatorname{SAE}^{(9)}$.

O Processo de Enfermagem é um instrumento metodológico e sistemático de prestação de cuidados, Que serve à atividade intelectual do enfermeiro e Que provê um guia para um determinado estilo de julgamento. Por ser um instrumento, seu uso pode ou não ser adeQuado e que ele por si só não é capaz de garantir a Qualidade da assistência(7).

Considera-se ainda, Que na literatura metodologia da assistência, processo de enfermagem e SAE tem sido utilizadas com o mesmo princípio organizador da assistência ${ }^{(10)}$.

Existem diferentes métodos utilizados para a obtenção da Qualidade na assistência de enfermagem, por meio de instrumentos como o processo de enfermagem. Alguns autores afirmam Que trabalhar a SAE por si só eleva a Qualidade da assistência ${ }^{(3,11,12)}$. Porém há Que se considerar Que não há estudo publicado na literatura nacional Que demonstre Que a implementação da SAE, no contexto dos hospitais brasileiros, garanta a Qualidade da assistência de enfermagem.

Outro dado relevante a ser considerado foi a resolução do Conselho Federal de Enfermagem (COFEN) de 2002, Que definiu a SAE como sendo atividade privativa do enfermeiro ${ }^{(3)}$. Consideramos Que tal afirmativa do COFEN deveria levar em consideração a participação dos demais membros da equipe de enfermagem, sobretudo na implementação dos cuidados, haja vista
Que o próprio COFEN define a SAE como atividade privativa do enfermeiro. Estaríamos aí configurando um nó deontológico? O grande impasse reside no fato de Que o gerenciamento, por meio da SAE, é atividade privativa do enfermeiro, porém algumas etapas da metodologia da assistência de enfermagem são de execução por todos os membros da equipe de enfermagem. Desta forma é importante diferenciar na resolução a execução do gerenciamento propriamente dito.

A segunda corrente Que trata Processo de Enfermagem e Metodologia da Assistência como sinônimos, pode ser abordada como a aplicação prática de uma teoria de enfermagem na prática assistencial, tratando-se de uma forma de organizada de cuidar do paciente segundo alguns passos estabelecidos ${ }^{(13,14)}$. Segundo essa abordagem o processo de enfermagem constitui um método a ser seguido e não de uma ferramenta a ser utilizada. Para justificar o uso do termo método, há contradição entre autoras: "o processo de enfermagem exige uma sistematização consciente do trabalho, a partir de uma metodologia adeQuada à produção do cuidado necessário"(9). Ou seja, o Processo de Enfermagem não constitui um método, ele prevê um método para ser implementado à prática.

O Processo de Enfermagem como um instrumento de trabalho, e também como um método sistematizador foi outra definição utilizada ${ }^{(15)}$.

Como se pode perceber existe um grupo de pesquisadoras que acreditam Que o processo é um método. Não Que essa afirmativa esteja incorreta, mas do ponto de vista semântico as concepções de processo (ato de proceder, de ir por diante) e de método (caminho para chegar a um determinado objetivo) são diferentes ${ }^{(16)}$.

A terceira corrente trata os três termos como sendo sinônimos. Descreve Que existem ainda outras terminologias para descrever a Metodologia da Assistência, tais como: o processo de enfermagem, o processo de cuidado, metodologia do cuidado, processo de assistir, consulta de enfermagem e sistematização da assistência de enfermagem ${ }^{(17,18)}$.

Nessa linha podemos afirmar Que atualmente existem muitos profissionais Que na prática acreditam na similaridade entre os termos. Existe inclusive um conflito ideológico Quando se traz à tona, a Questão do vocabulário padronizado para o registro de enfermagem. Na prática muitos enfermeiros desconhecem esses vocabulários e abordam os mesmos como se fossem métodos de assistência de enfermagem, chegando a confundi-los com o próprio processo de enfermagem por assim dizer.

Grande parte dos vocabulários elaborados para o registro de informações na área da saúde podem ser agrupados em Quatro categorias distintas: terminologias, nomenclaturas, classificações e thesaurus ${ }^{(19)}$ :

Classificação: sistema onde entidades específicas são agrupadas de acordo com critérios de similaridade, sendo esses códigos prédefinidos.

Nomenclaturas: códigos são atribuídos a conceitos médicos Que são listados podendo sofrer recombinações e assim serem reordenados por nomes ou conceitos, organizadas semântica ou hierarQuicamente.

Terminologias: conjunto de termos especializados combinados.

Thesaurus: lista de termos, Que contempla seus sinônimos, usados em uma determinada área de domínio; com o objetivo de padronizar termos ${ }^{(20)}$. 
Dentre algumas terminologias desenvolvidas para apoiar o registro e análise de enfermagem: a Classificação Clínica dos Cuidados - CCC; a CIPE (Classificação Internacional para a Prática de Enfermagem); a classificação de diagnósticos de enfermagem da NANDA (North American Nursing Diagnosis Association); a classificação das intervenções de enfermagem - NIC (Nursing Interventions Classification); a classificação dos resultados de enfermagem - NOC (Nursing Outcomes Classification); o conjunto de dados mínimos do cuidado ao paciente - PCDS (Patient Care Data Set) e o léxico e terminologia para intervenções de enfermagem - NILT (Nursing Interventions Lexicon Terminology)(21).

Atualmente no Brasil a CIPE e as classificações NANDA, NIC e NOC têm sido mais utilizadas, segundo constatação de suas aplicações em pesQuisas recentes ${ }^{(15,22-25)}$.

\section{DISCUSSÃO}

Ainda Que a literatura publicada sobre a SAE, apresente crescimento gradual desde 2000, a mesma ainda denota uma profunda disparidade de conceitos Que são próprios da prática profissional de enfermagem. Isso se traduz na prática por um enfraquecimento e uma desarticulação da teoria com a prática, fato este Que gera conflitos ideológicos Que prejudicam não somente o entendimento da prática de enfermagem, como também o ensino das teorias de enfermagem, bem como do processo de enfermagem e da metodologia da assistência.

Cabe ressaltar ainda Que existem poucos artigos Que relacionem a sistematização da assistência de enfermagem com as atividades gerenciais desenvolvidas pelo enfermeiro; a maior parte dos artigos trata de implementação de metodologias assistenciais à prática.

Diante do Que a literatura aborda, a primeira corrente de pensamento abarca as idéias Que corroboramos; onde a SAE, o Processo de Enfermagem e a Metodologia da Assistência são encarados como conceitos distintos. Isso porQue a sistematização da assistência demanda alguma ferramenta a ser utilizada e as ferramentas para sistematização demandam métodos de trabalho, previstos nas metodologias para a assistência de enfermagem.

Nesse sentido a SAE situa-se numa esfera institucional, onde a visão de organização das atividades de enfermagem interfere diretamente na implantação e implementação do processo de enfermagem e por assim dizer na metodologia subsidiada em uma teoria de enfermagem a ser utilizada na prática. Nesse sentido cabe destacar ainda que o processo de enfermagem é único, o que pode se modificar à luz do referencial teórico da unidade, é a metodologia da assistência de enfermagem proposta por cada uma das Teóricas de Enfermagem para o trabalho processual.

É importante considerar Que a implementação da SAE não se dá apenas através do processo de enfermagem, ela pode ocorrer por meio de outras ferramentas tal como a consulta de enfermagem. Ainda assim far-se-á necessário o emprego de algum método para sistematizar a assistência, onde cada cenário de aplicação utilizese da metodologia mais adequada a sua realidade; baseada na teoria de enfermagem Que irá nortear a prática da enfermagem. A escolha de um método é essencial, e há Que se considerar Que não existe um método Que represente um padrão para as unidades de saúde e nem tampouco para a garantia da Qualidade da assistência. Sabe-se que existem diferentes métodos e realidades. Daí encontrar a equação ideal entre ambos, visando a Qualidade da assistência, representa hoje um grande desafio ao gerenciamento de enfermagem.

Sobre as terminologias de enfermagem há Que se considerar Que são tentativas de uniformizar a linguagem utilizada pela enfermagem, mas a implementação das mesmas não prevê fases como num método de trabalho. As terminologias de enfermagem propõem estruturas classificatórias para diferentes etapas da metodologia da assistência. Considera-se ainda Que o emprego das mesmas não é obrigatório, podendo cada unidade eleger a terminologia Que melhor retrate a realidade de sua clientela. Ainda Que não haja obrigatoriedade no emprego, cabe considerar Que a enfermagem necessita discutir e aplicar suas terminologias com fins de crescimento e sustentação da prática profissional. Prática essa pautada no raciocínio clínico e crítico, bem como para acompanhar movimentos internacionais de padronização da linguagem de enfermagem tal como o da Classificação Internacional para a Prática de Enfermagem (CIPE), defendida no âmbito internacional pelo International Council of Nursing (Conselho Internacional de Enfermagem) e na esfera nacional pela Associação Brasileira de Enfermagem (ABEn).

\section{CONSIDERAÇÕES FINAIS}

Diante dos achados da literatura fica evidente a necessidade de uma uniformização da linguagem empregada no Que se refere a SAE. Tamanha disparidade demonstra Que ainda Que o enfermeiro busque se apropriar de conceitos da sua prática, ele pode ainda apresentar dúvidas Que certamente podem impactar na aplicação de ferramentas como o processo de enfermagem à prática.

A literatura demonstra o crescimento de iniciativas de implementação de metodologias assistenciais, mas ainda carece de uma maior articulação teórico-prática a fim de Que a SAE seja encarada como elemento fortalecedor da identidade profissional.

Há que se considerar a freqüente necessidade de discussão sobre a temática a fim de buscarmos um consenso na linguagem Que promova a Qualificação e aprimoramento da enfermagem.

\section{REFERÊNCIAS}

I. Tronchin DMR, Melleiro MM, Takahashi RT. A Qualidade a avaliação dos serviços de saúde e de enfermagem. In: Kurgcgant P, organizadora. Gerenciamento em enfermagem. Rio de Janeiro: Guanabara Koogan; 2005.

2. Murahovschi D. Curso Talsa Multiplicadores. [citado em: 1 I nov 2007]. Disponível em http://www.ona.org.br

3. Conselho Federal de Enfermagem (BR). Resolução COFEN No
272/2002. Dispõe sobre a Sistematização da Assistência de Enfermagem - SAE - nas Instituições de Saúde Brasileiras. [citado em: 15 jan 2007]. Disponível em: http:// www.portalcofen.gov.br/2007/materias.asp?ArticleID = $7 \mid 00 \&$ section $I D=34$

4. Fuly PSC, Freire SM, Almeida RT. The Nursing Process and its Application in Intensive Care at Rio de Janeiro as a Support to 
the Development of an Electronic Patient Record. Online Braz I Nurs 2003; 2(3).

5. Conselho Regional de Enfermagem São Paulo. Decisão COREN-SP/DIR/008/99. "Normatiza a Implementação da Sistematização da Assistência de Enfermagem - SAE - nas Instituições de Saúde, no âmbito do Estado de São Paulo." [citado em: 15 jan 2007]. Disponível em: http:// www.corensp.org.br/072005/noticias/revista/anteriores/ janeiro_fevereiro00/7.htm

6. Aquino DR, Filho WDL. Construção da Prescrição de Enfermagem Informatizada em uma UTI. Cogitare Enferm 2004; 9 (I): 60-70.

7. Cruz DALM. Processo de enfermagem e classificações. In: Gaidzinski RR. Diagnóstico de enfermagem na prática clínica. Porto Alegre: Artmed; 2008.

8. Backes DS, Schwartz E. Implemetação da Sistematização da Assistência de Enfermagem: Desafios e ConQuistas do Ponto de Vista Gerencial. Rev Ciência Cuidado Saúde 2005; 4(2): 182-8.

9. Leopardi MT. Teoria e método em assistência de enfermagem. $2^{\mathrm{a} e d .}$. Florianópolis: Soldasof; 2006.

10. Mendes MA, Bastos MAR. Processo de Enfermagem: seQüências no cuidar, fazem a diferença. Rev Bras Enferm 2003; 56(3): 27I-6.

1 1. Bittar DB, Pereira LV, Lemos RCA. Sistematização da Assistência de Enfermagem ao Paciente Crítico: proposta de instrumento de coleta de dados. Texto Contexto Enferm 2006; 1 5(4): 617 28.

12. Cunha SMB, Barros ALBL. Análise da Implementação da Sistematização da Assistência de Enfermagem, Segundo o Modelo Conceitual de Horta. Rev Bras Enferm 2005; 58(5): 568-72.

13. Hermida PMV. Desvelando a Implementação da Sistematização da Assistência de Enfermagem. Rev Bras Enferm 2004; 57(6): 733-7.

14. Tannure MC. O Processo de enfermagem. In: Tannure MC,
Gonçalves AMP. Sistematização da assistência de enfermagem: guia prático. Rio de Janeiro: Guanabara Koogan; 2008.

15. Brandalize DL, Kalinowski CE. Processo de Enfermagem: vivência na implantação da fase de diagnóstico. Cogitare Enferm 2005; 10(3): 53-7.

16. Ferreira $\mathrm{ABH}$. Novo dicionário Aurélio da língua portuguesa. Rio de Janeiro: Positivo Editora; 2004.

17. Andrade IS, Vieira MJ. Prática Assistencial de Enfermagem: problemas, perspectivas e necessidade de sistematização. Rev Bras Enferm 2005; 58(3): 26I-5.

18. Carraro TE, Kletemberg DF., Gonçalves, L.M. O Ensino da metodologia da Assistência de Enfermagem no Paraná. Rev Bras Enferm 2003; 56(5): 499-501.

19. Amaral MB. Padrões de registro e transmissão de dados em saúde. In: Massad E, Marin HF, Azevedo RS. O prontuário eletrônico do paciente na assistência, informação e conhecimento médico. São Paulo: USP/UNIFESP; 2003.

20. Bemmel IA, Mussen MA. Handbock of medical informatics. Stuttgart: Springer Verlag; 1997.

21. Marin HF. Os componentes de enfermagem do prontuário eletrônico do paciente. In: Massad E, Marin HF, Azevedo RS. O prontuário eletrônico do paciente na assistência, informação e conhecimento médico. São Paulo: USP/UNIFESP; 2003.

22. Nóbrega MML, Garcia TR. Perspectivas de incorporação da Classificação Internacional para a Prática de Enfermagem. Rev Bras Enferm 2005; 58(2): 227-30.

23. Vale IN, Lopes MHBM. A Utilização de classificações na prática e no ensino de enfermagem: a experiência da Unicamp. Rev Bras Enferm 2002; 55(6): 670-3.

24. Moreira LFR, Ferreira LS, Chianca TCM. Experiência discente de Sistematização da Assistência junto a membros de uma equipe de enfermagem de um Hospital-Escola. REME 2004; 8(I): 235-40.

25. Nóbrega LRB. Sistematização da Assistência de Enfermagem na unidaden neurocirúrgica: levantamento de diagnósticos de enfermagem. Acta Paul Enferm 2000; 13(esp-II): 91-3. 